Gazi University
Journal of Science
http://dergipark.gov.tr/gujs

\title{
Direct Displacement Based Design for Reinforced Concrete Framed Structures with Seismic Isolation
}

\author{
Channabasaveshwar CHIKMATH* (D) , Ankit SODHA (D) Sandip VASANWALA (D) \\ Civil Engineering Department, Sardar Vallabhbhai National Institute of Technology, 395007, Surat, India
}
Highlights
- RC structures of four, eight and twelve storey are analyzed by Direct Displacement Based Design.
- Nonlinear time history analysis is performed for the ground motions according to fault distance.
- Seismic isolators in the form of Lead Rubber Bearing are used.

Article Info

Received: 16 Nov 2020

Accepted: 01 June 2021

\section{Keywords}

Direct displacement based design Nonlinear time history analysis Hysteretic damping Lead rubber bearing

\begin{abstract}
Direct displacement-based design is a nonlinear static procedure and has to check the suitability of the method against different types of ground motions namely far field, near field forward directivity and near field fling step. The method is applied for the buildings supported on a fixed base and hysteretic isolation bearings. Seismic isolators are provided between the foundation and the superstructure to minimize the influence of ground motion on the superstructure. The method is applied for four, eight and twelve storey reinforced concrete frame structures equipped with and without seismic isolators. Lead rubber bearing is used as seismic isolators. An equivalent damping ratio, derived from the particular characteristics of buildings supported on isolation bearings, is suggested. The energy dissipation mechanism in the isolators controls the displacement of the structure within acceptable limits at the level of the isolator. The results were validated with nonlinear time history analysis and were found to be in good agreement with the Direct displacementbased design methodology for far field ground motions. The performance of the building was measured for interstorey drift ratio, time period, acceleration of top floor, base shear, isolator displacement. This is an attempt to link the direct displacement-based design of the reinforced concrete building with seismic isolators subjected to the far field, near field forward directivity, near field fling step ground motions.
\end{abstract}

\section{INTRODUCTION}

Damages induced in the structure after an earthquake are measured in terms of displacements, drifts, rotations which arises the concept of Displacement-based design which is more appealing than the traditional Force-based design method that uses forces and stresses as the input parameter. The displacement-based design has multi-performance levels to achieve the desired performance limit based on the type of the structure. Ductility is also as important as strength was realized in the 70's [1]. The first generation performance-based design procedures [2-5] laid the fundamental concepts of displacementbased design. The goal of SEAOC Vision 2000 is to develop the framework for procedures that lead to the design of structures of predictable seismic performance and can incorporate multiple performance objectives to achieve the stated performance levels for the given hazard levels [5]. Applied Technology Council emphasizes the use of the capacity spectrum method which involves determining the capacity and demand spectra. ATC 40 is limited to concrete buildings only. Although the capacity spectrum method is simple, the theoretical basis and physical interpretations are in a debatable stage [2]. FEMA 273 includes different performance objectives with associated ground motions. Analysis and design methods for the multi-level performance range from linear static to inelastic time history analysis. Drift limits for various lateral load resisting systems at different performance levels were proposed [3]. The FIB CEB-FIP [6] formulated the procedure for the displacement-based design of RC structures for Euro code [7]. 
Direct Displacement Based Design (DDBD) is a displacement-based design method developed by Priestly [8] on reinforced concrete frame buildings. The method introduces the use of two different deformed shapes for buildings less than or equal to four storey and for buildings greater than four storey, hysteretic damping in addition to viscous damping in the procedure and an expression for higher modes in tall structures was proposed. The results were validated using time history analysis of various earthquake intensity ranges. The results obtained by the equations proposed by Priestley were in good agreement with time history analysis. Different structural systems namely moment-resisting frame, wall frame and steel braced systems with the direct displacement-based design were analyzed and validated by nonlinear time history analysis which proved that the DDBD approach was efficient [9]. Direct displacement-based design in two and three-bay reinforced concrete structures was carried out considering plastic hinge length, longitudinal reinforcement ratio in the members [10]. The expression for considering higher modes in direct displacement-based design for vertically irregular moment-resisting frames was put forward and verified with nonlinear time history analysis [11]. An effort was made to simplify the direct displacement-based design method using viscous dampers [12]. Two displacement-based methods namely direct displacementbased design and displacement-based seismic design method using damage control were applied on a twelve storeys plan irregular RC frame building [13]. The latter one gave a better result but still needs to be validated considering the different configuration of the building. A performance-based assessment tool known as Displacement-based adaptive pushover analysis was applied for concrete frame buildings varying in height [14]. Seismic performance of steel moment resisting frame was carried on four, six and storey with mass irregularity [15].

Ground motion records are of two types, based on the distance of the recording station from the fault site namely far field $(>15 \mathrm{~km})$ and near field $(<15 \mathrm{~km})[16]$. Far field ground motions have large amplitude and longer duration. The directivity effect and fling-step effect are the two paramount effects associated with near-field earthquakes. In forward directivity, rupture propagation is aligned to the site and fault rupture velocity is approximately equal to the shear wave velocity of the site [17]. This results in large amplitude, long period and short duration. The permanent ground displacement due to tectonic deformations causes the fling step effect. It produces large amplitude velocity pulse. Diagrammatic representation of the far field, forward directivity and fling step is shown in Figure 1.
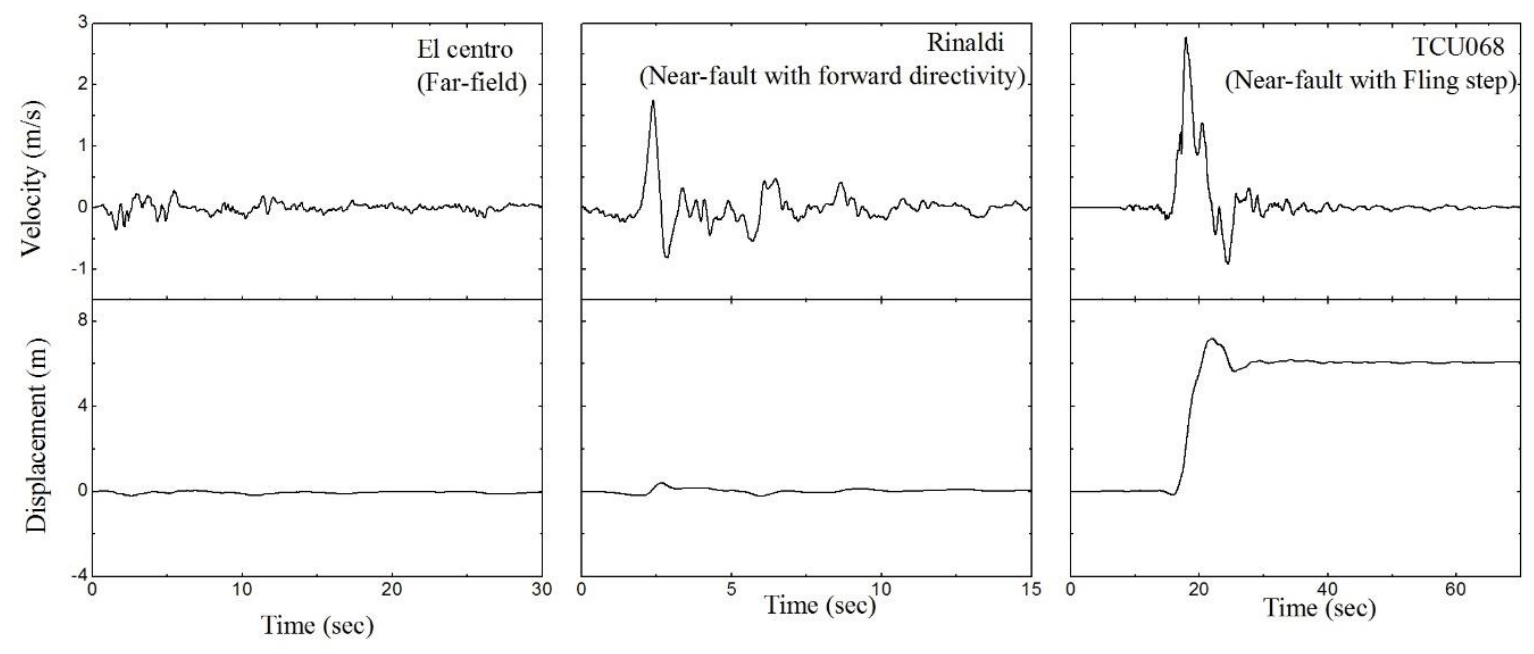

Figure 1. Comparison of far field, forward directivity and fling step ground motions

The seismic response of six storey and thirteen storey buildings due to fling step and forward directivity were determined [17]. Moghim et al., [18] applied direct displacement-based design for concrete buildings situated in near fault regions. Alternate to the Gutenberg-Richter model, the interrelation between the 
number of earthquakes and their magnitudes was proposed by considering 4863 ground motions of magnitude 4.0 and above [19].

The term responsive index was coined to compare and achieve the stated performance levels in fixed and base-isolated structure [20]. The effect of the behaviour of the base-isolated ten storey building with and without the shear wall, yielded large peak storey drift in moment resisting frames than the shear wall building [21]. Bhandari et al., [22] worked on ten storey base-isolated RC building frame subjected to the far field, near field forward directivity and near field fling step ground motions of design base earthquake(DBE) and maximum considered earthquake(MCE) in terms of base shear, floor acceleration, interstorey drift, isolator displacement and the number of hinges formed. Lead rubber bearing was used as the isolator. Hallow rubber bearing was found more efficient when compared to solid rubber bearing since the stiffness is reduced in the former when compared to the latter [23]. Rubber bearings and dampers were found effective in reducing the damage due to earthquake on the steel liquid storage tank [24].

Although seismic base isolation and displacement-based design were developed in the 70s only, the displacement-based design with the base isolation on bridges was applied in 2008 [25] and on buildings in 2010 [26]. Cardone et al., [26] modified the direct displacement-based design developed by Priestley [8] for the different types of base-isolated frame structures.

In this work, the direct displacement-based design procedure developed by Cardone et al., [26] is applied on fixed base(FB) and base-isolated(BI) building of four, eight and twelve storey RC frame buildings considered as low-rise, medium-rise and high-rise buildings $[9,27]$ respectively which form the major frame buildings in India located in Zone-V, medium soil of Indian seismic code [28] subjected to the far field, near field forward directivity and near field fling step ground motions. Seismic isolator in the form of Lead rubber bearing (LRB) is used. An equivalent damping ratio, derived from the particular characteristics of buildings supported on isolation bearings, is suggested. The energy dissipation mechanism in the isolators controls the displacement of the structure within acceptable limits at the level of the isolator. The mechanical properties of the isolator are derived after literature review and selected after a thorough examination of the product catalogue available on the manufacturer's website [29-32]. The performance of the buildings is measured concerning inter-storey drift ratio, roof acceleration, base shear, isolator displacement. The buildings are designed by the Indian concrete code [33].

\section{PROCEDURE}

In this method, a multi-degree of freedom system is represented into an equivalent single degree with effective mass $m_{e}$ and effective height $h_{e}$ as shown in Figure $2 . K_{e}$ is the secant stiffness of the system at the ultimate displacement of this system shown in Figure 3. The procedure is as follows:
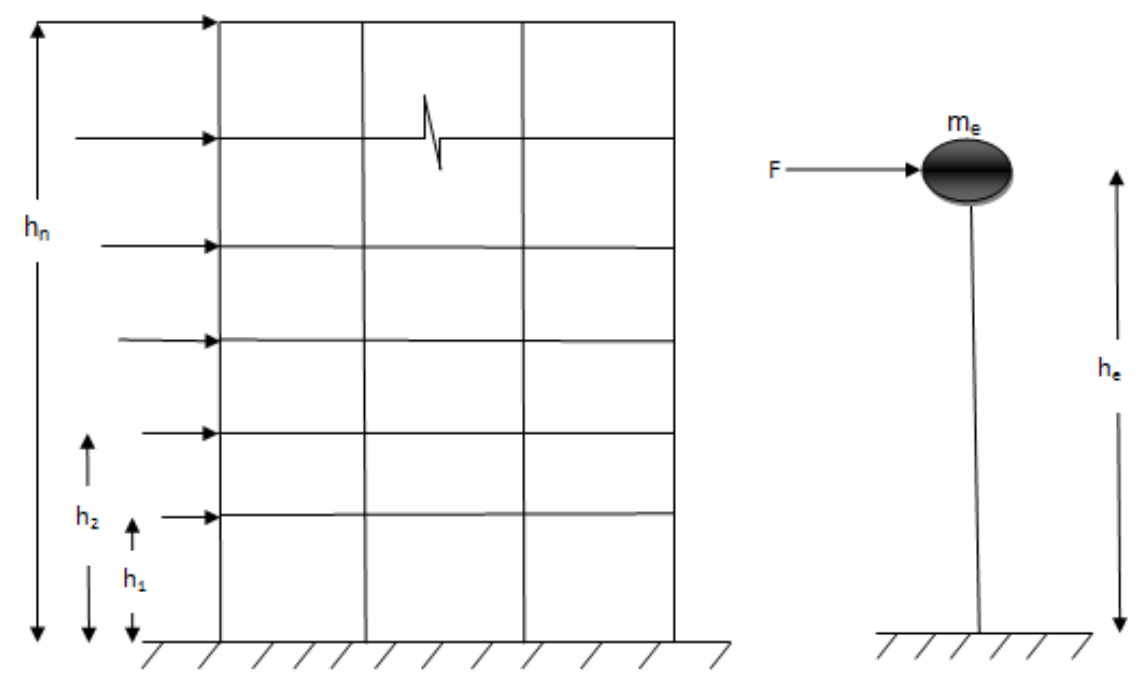

Figure 2. Simulation of MDOF to SDOF 


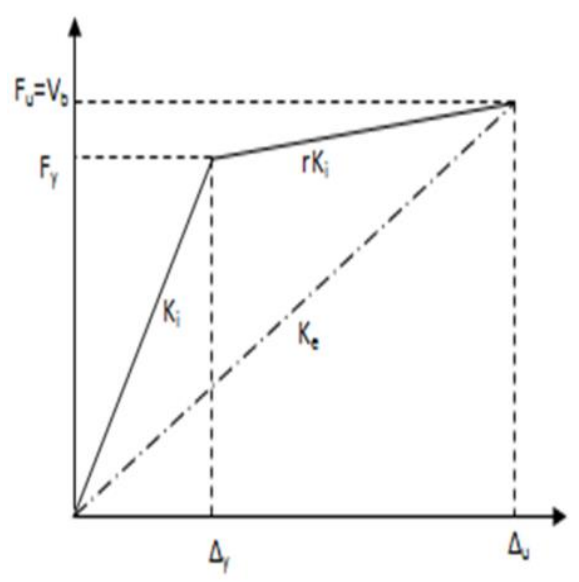

Figure 3. Effective stiffness

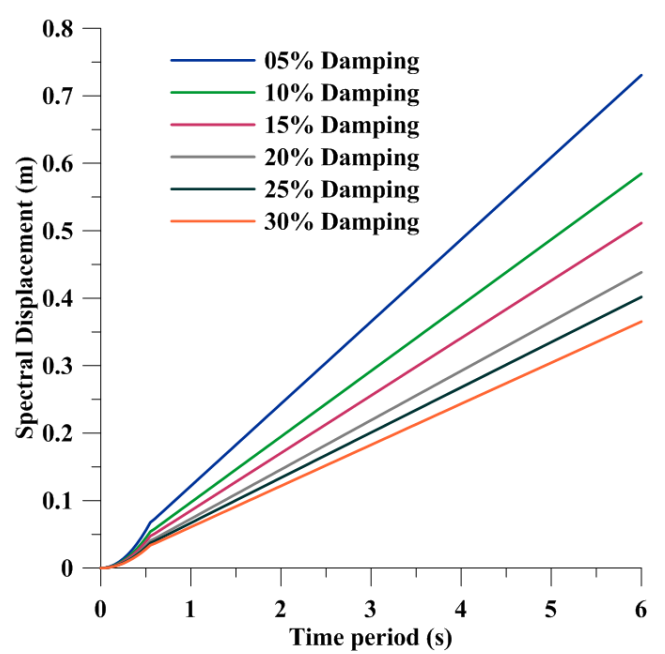

Figure 4. Displacement spectrum for IS 1893-2016 for Zone- V

1. The first mode deformed shape is derived using the expression [26] given by

$$
\Phi_{i}=\cos \left[\left(\frac{1}{I_{r}}\right) \cdot\left(1-\frac{h_{i}}{h_{n}}\right) \cdot \frac{\pi}{2}\right]-\cos \left[\left(\frac{1}{I_{r}}\right) \cdot \frac{\pi}{2}\right]
$$

where $h_{i}=$ height of $i^{\text {th }}$ storey from the base, $h_{n}=$ total height of the structure, $I_{r}$ is the ratio of the effective period of vibration of seismically isolated structure to fundamental period of vibration of fixed support building. For fixed supported building, $\mathrm{I}_{\mathrm{r}}=1$.

2. Select the appropriate base isolator with the isolator displacement $D_{d}$ and maximum interstorey drift ratio $\theta_{\mathrm{d}}$. The critical storey is the storey where the maximum interstorey drift ratio is reached. The maximum interstorey drift ratio is reached in the first storey [13] and is assumed as

$$
\theta_{d}=100 \frac{\Delta_{c}}{h_{c}} .
$$

3. The displacement profile [26] for the $\mathrm{i}^{\text {th }}$ storey of the structure is given as

$$
\begin{gathered}
\Delta_{i}=D_{d}+\theta_{d} \cdot c_{1} \cdot \Phi_{i}, \\
c_{1}=\frac{h_{1}}{100 \Phi_{1}} .
\end{gathered}
$$


4. Design displacement $\Delta_{\mathrm{d}}$, effective mass $\mathrm{m}_{\mathrm{e}}$, the effective height $\mathrm{h}_{\mathrm{e}}$ of equivalent SDOF system are given by Equations (5)-(7), respectively

$$
\begin{gathered}
\Delta_{d}=\frac{\sum_{i=1}^{n} m_{i} \Delta_{i}^{2}}{\sum_{i=1}^{n} m_{i} \Delta_{i}}, \\
m_{e}=\frac{\sum_{i=1}^{n} m_{i} \Delta_{i}}{\Delta_{d}}=\frac{\left[\sum_{i=1}^{n} m_{i} \Delta_{i}\right]^{2}}{\sum_{i=1}^{n} m_{i} \Delta_{i}{ }^{2}}, \\
h_{e}=\frac{\sum_{i=1}^{n} m_{i} \Delta_{i} h_{i}}{\sum_{i=1}^{n} m_{i} \Delta_{i}} .
\end{gathered}
$$

5. Since displacement, drift, ductility are the governing parameters in the displacement-based design method, design ductility can be controlled as

$$
\begin{gathered}
\mu_{d}=\frac{\theta_{d}}{\theta_{y}}, \\
\theta_{y}=0.5 \varepsilon_{y} \frac{l_{b}}{h_{b}},
\end{gathered}
$$

$\varepsilon_{\mathrm{y}}=$ yield strain in steel $=0.2 \%, \mathrm{l}_{\mathrm{b}}=$ beam length and $\mathrm{h}_{\mathrm{b}}=$ beam depth.

6. In addition to $5 \%$ elastic viscous damping, hysteretic damping is added to include energy dissipation by $\mathrm{RC}$ members during the earthquake and is known as equivalent viscous damping of the superstructure [13]

$$
\begin{gathered}
\xi_{S}=\left(5+\xi_{\text {hyst }}\right) \% \\
\xi_{S}=5+120\left(\frac{1-\mu_{d}^{-0.5}}{\pi}\right) \% .
\end{gathered}
$$

7. The equivalent damping ratio $\xi_{\text {eq }}$ of base-isolated structure is the combination of damping ratios of superstructure and base isolator at their corresponding displacements given as

$$
\xi_{e q}=\frac{\left[\xi_{I S} \cdot D_{d}+\xi_{S} \cdot\left(\Delta_{D}-D_{d}\right)\right]}{\Delta_{D}} .
$$

8. The effective time period $\mathrm{T}_{\mathrm{eq}}$ is established by entering the displacement spectra set shown in Figure 4 with the design displacement $\Delta_{\mathrm{d}}$ and the equivalent viscous damping.

9. The equivalent stiffness $\mathrm{K}_{\mathrm{eq}}$ for the design displacement of the equivalent SDOF system using

$$
K_{e q}=4 \pi^{2} \frac{m_{e}}{T_{e q}^{2}} .
$$

10. The base shear is the product of equivalent stiffness $K_{\text {eq }}$ and the design displacement $\Delta_{\mathrm{d}}$, given as

$$
V_{b(D D B D)}=K_{e q} \Delta_{d} \text {. }
$$

11. Distribute the base shear along the height of the building using Equation (14)

$$
F_{i}=V_{b(D D B D)} \cdot \frac{m_{i} \Delta_{i}}{\sum_{i=1}^{n} m_{i} \Delta_{i}} .
$$

12. The stiffness of the base isolator is given by

$$
K_{I S}=\frac{V_{b(D D B D)}}{D_{d}} .
$$




\section{CHARACTERISTICS OF LEAD RUBBER BEARING}

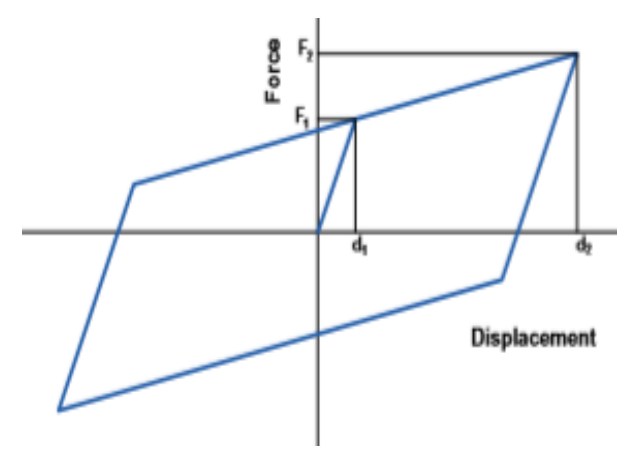

Figure 5. Hysteresis loop for LRB

The hysteresis loop is typically modelled as bilinear for a lead rubber bearing isolator. The parameters $F_{1}$, $\mathrm{d}_{1}, \mathrm{~F}_{2}$ and $\mathrm{d}_{2}$ that define the bilinear curve are given by the manufacturers for each standard LRB [29-32]. The hysteretic behaviour of an LRB can also be modelled as linear, using the effective stiffness $K_{e}$ and the equivalent viscous damping coefficient $\xi_{I S}$, which depends on the maximum displacement $\mathrm{d}_{2}$ and the corresponding force $\mathrm{F}_{2}$, to which refer to Figure 5.

$$
\begin{gathered}
K_{e}=\frac{F_{2}}{d_{2}} \\
\xi_{I S}=\frac{2}{\pi}\left[\frac{F_{1}}{F_{2}}-\frac{d_{1}}{d_{2}}\right]
\end{gathered}
$$

\section{CHARACTERISTICS OF GROUND MOTIONS}

Ground motion data of six set each for the far field, near field forward directivity and near field fling step respectively have been selected [34]. The details of the ground motions in terms of magnitude, recording station, PGA, fling step displacement are shown in Table 1.

\begin{tabular}{|c|c|c|c|c|c|}
\hline Record Label & Earthquake & Magnitude & Station & PGA (g) & Fling Disp. $(\mathrm{cm})$ \\
\hline \multicolumn{6}{|c|}{ Far-field ground motions } \\
\hline FF 1 & 1999 Chamoli & 6.4 & Chamoli & 0.359 & - \\
\hline FF 2 & 1940 Imperial Valley & 6.95 & El Centro & 0.313 & - \\
\hline FF 3 & 1989 Loma Prieta & 6.9 & Capitola & 0.420 & - \\
\hline $\mathrm{FF} 4$ & 1994 Northridge & 6.7 & Northridge-Saticoy & 0.529 & - \\
\hline FF 5 & 1994 Northridge & 6.7 & Canoga Park & 0.477 & - \\
\hline FF 6 & 1987 Superstition Hills & 6.7 & El Centro Imp Co. Centre & 0.512 & \\
\hline \multicolumn{6}{|c|}{ Near-fault ground motions with forward directivity } \\
\hline NFD 1 & 1994 Northridge & 6.7 & Rinaldi & 0.890 & - \\
\hline NFD 2 & 1994 Northridge & 6.7 & Sylmar & 0.730 & - \\
\hline NFD 3 & 1994 Northridge & 6.7 & Newhall & 0.720 & - \\
\hline NFD 4 & 1979 Imperial Valley & 6.4 & EL Centro Array 7 & 0.460 & - \\
\hline NFD 5 & 1992 Landers & 7.3 & Lucerne Valley & 0.710 & - \\
\hline NFD 6 & 1979 Imperial Valley & 6.7 & EL Centro Array 5 & 0.370 & \\
\hline \multicolumn{6}{|c|}{ Near-fault ground motions with fling step } \\
\hline NFS1 & 1999 Chi Chi & 7.6 & TCU129_NS & 0.610 & 67.54 \\
\hline NFS 2 & 1999 Chi Chi & 7.6 & TCU084_NS & 0.420 & 59.43 \\
\hline NFS 3 & 1999 Chi Chi & 7.6 & TCU074 EW & 0.590 & 174.56 \\
\hline NFS4 & 1999 Chi Chi & 7.6 & TCU052_NS & 0.440 & 697.12 \\
\hline NFS5 & 1999 Chi Chi & 7.6 & TCU068_EW & 0.500 & 601.84 \\
\hline NFS 6 & 1999 Kocaeli & 7.4 & $\mathrm{YPT}^{-}$ & 0.23 & 145.79 \\
\hline
\end{tabular}

Table 1. Ground motion records 


\section{BUILDING DETAILS}

The plan of an RC frame building with dimensions is shown in Figure 6 for four, eight and twelve storeys respectively. The building is located in medium soil for the Bhuj area which falls under Zone-V considered an extreme zone according to the Bureau of Indian Standards [28]. The design is carried out using the Bureau of Indian Standard code [33]. The drift is limited to 2\% [5,10]. Nonlinear time history analysis (NLTHA) is carried out to verify the inter-story drift ratio, acceleration of top floor, base shear, isolator displacement for six sets of each earthquake ground motions of far field, near field forward directivity and near field fling step respectively scaled to Zone-V [28] using with FB and BI. The analysis and design were carried out in MIDAS/GEN 2019 software. The live load is $3 \mathrm{kN} / \mathrm{m}^{2}$. External wall of $230 \mathrm{~mm}$ thick exists. The thickness of the slab is $150 \mathrm{~mm}$. Characteristic strength of main steel and secondary steel are $500 \mathrm{~N} / \mathrm{mm}^{2}$ and $415 \mathrm{~N} / \mathrm{mm}^{2}$ respectively. Secondary beams are $300 \mathrm{~mm}$ in width and $450 \mathrm{~mm}$ in depth. The dimensions of the members are as shown in Table 2. The lead rubber bearing provided is manufactured by FIP INDUSTRIALE [31]. The isolators were selected based on the maximum isolator displacement, a good separation of the time period for fixed and base-isolated structure and axial load coming on the columns $[22,35]$. Isolator properties are shown in Table 3.

Table 2. Properties of frame building

\begin{tabular}{|c|c|c|c|c|c|}
\hline & Member & Floor & Width (mm) & Depth (mm) & $\begin{array}{c}\text { Grade of concrete } \\
\text { cube }\left(\mathrm{N} / \mathrm{mm}^{2}\right)\end{array}$ \\
\hline \multirow{2}{*}{ 4-Storey } & Beam & \multirow{2}{*}{$1-4$} & 300 & 600 & \multirow{2}{*}{30} \\
\hline & Column & & 550 & 550 & \\
\hline \multirow{2}{*}{ 8-Storey } & Beam & \multirow{2}{*}{$1-8$} & 300 & 600 & \multirow{2}{*}{30} \\
\hline & Column & & 650 & 650 & \\
\hline \multirow{2}{*}{ 12-Storey } & Beam & \multirow{2}{*}{$1-12$} & 300 & 600 & \multirow{2}{*}{30} \\
\hline & Column & & 750 & 750 & \\
\hline
\end{tabular}

Table 3. Isolator properties

\begin{tabular}{llll}
\hline & 4 Storey & 8 Storey & 12 Storey \\
\hline Name of the isolator & LRB-S 550/200-120 & LRB-S 550/200-120 & LRB-S 600/204-130 \\
Isolator Displacement $D_{\mathrm{d}}$ & $0.4 \mathrm{~m}$ & $0.4 \mathrm{~m}$ & $0.4 \mathrm{~m}$ \\
Effective Stiffness Keff $_{\text {eft }}$ & $810 \mathrm{kN} / \mathrm{m}$ & $810 \mathrm{kN} / \mathrm{m}$ & $950 \mathrm{kN} / \mathrm{m}$ \\
Initial Stiffness K & $7875 \mathrm{kN} / \mathrm{m}$ & $7875 \mathrm{kN} / \mathrm{m}$ & $9250 \mathrm{kN} / \mathrm{m}$ \\
Effective Damping $\xi_{\mathrm{IS}}$ & $26.65 \%$ & $26.65 \%$ & $26.85 \%$ \\
Post Yield Stiffness Ratio $\gamma$ & 0.058 & 0.058 & 0.057 \\
Yield Force Fy & $126 \mathrm{kN}$ & $126 \mathrm{kN}$ & $148 \mathrm{kN}$ \\
Vertical Stiffness $\mathrm{K}_{\mathrm{v}}$ & $789000 \mathrm{kN} / \mathrm{m}$ & $789000 \mathrm{kN} / \mathrm{m}$ & $844000 \mathrm{kN} / \mathrm{m}$ \\
\hline
\end{tabular}



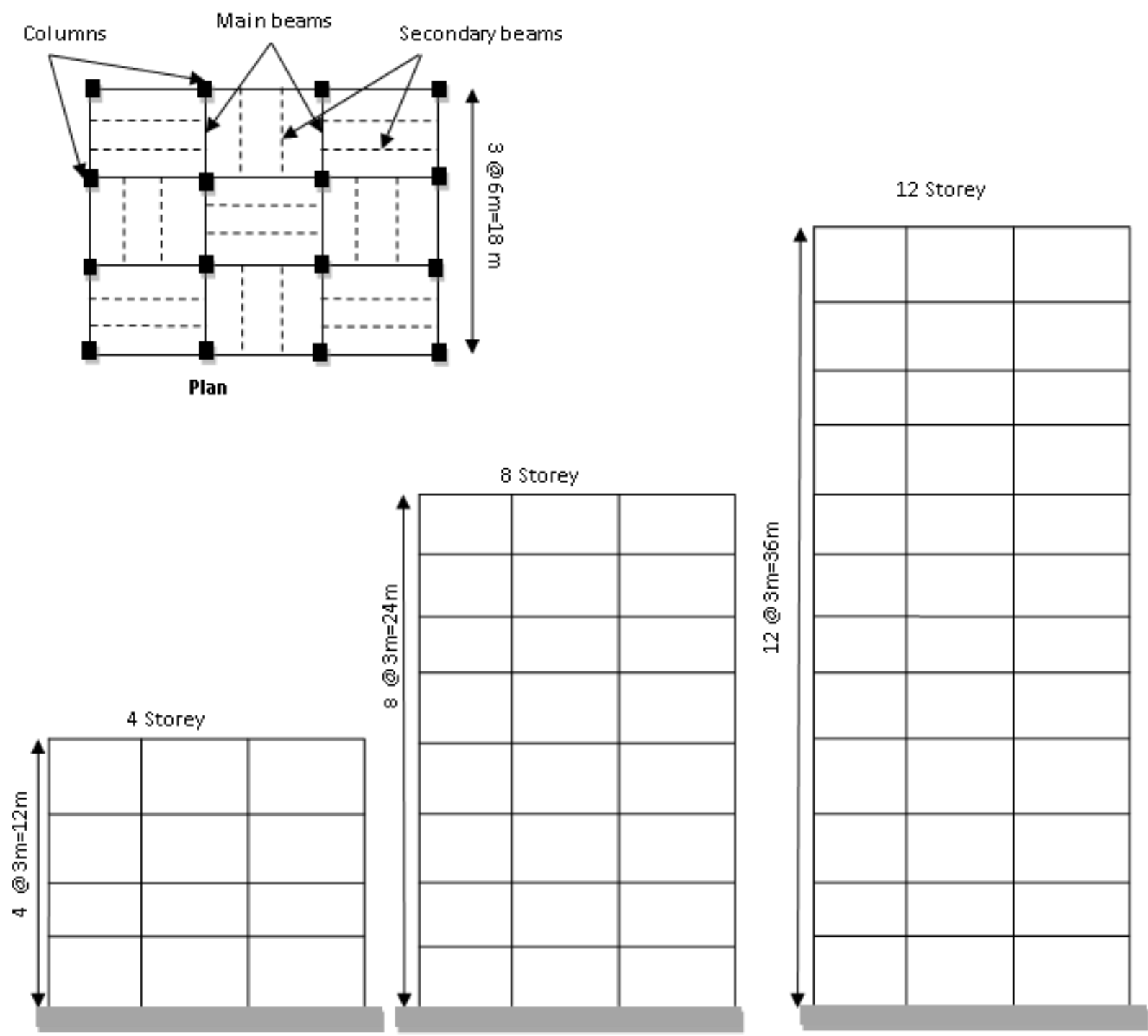

Elevation

Figure 6. Geometry of the building

Table 4. Results of analysis

\begin{tabular}{ccccccc}
\hline No of storeys & $\mathrm{T}_{\mathrm{eq}}(\mathrm{s})$ & $\mathrm{I}_{\mathrm{r}}$ & \multicolumn{2}{c}{$\xi_{\mathrm{eq}}(\%)$} & \multicolumn{2}{c}{$\mathrm{V}_{\mathrm{b}(\mathrm{DDBD})}(\mathrm{kN})$} \\
\hline & & & $\mathrm{BI}$ & $\mathrm{FB}$ & $\mathrm{BI}$ & $\mathrm{FB}$ \\
4 & 5.30 & 3.39 & 24.70 & 16.19 & 1237 & 3524 \\
8 & 5.70 & 2.43 & 23.11 & 16.19 & 2621 & 3554 \\
12 & 6.08 & 2.05 & 22.17 & 16.19 & 4116 & 4136 \\
\hline
\end{tabular}


Table 5. Four storey response parameters

\begin{tabular}{|c|c|c|c|c|c|c|}
\hline Earthquake & Type of structure & $\begin{array}{c}\text { Roof accel } \\
\left(\mathrm{m} / \mathrm{s}^{2}\right)\end{array}$ & $\begin{array}{c}\text { Max Drift ratio } \\
(\%)\end{array}$ & $\begin{array}{c}\text { Roof disp } \\
(\mathrm{mm})\end{array}$ & $\begin{array}{l}\text { first floor disp } \\
(\mathrm{mm})\end{array}$ & $\begin{array}{c}\text { Isolator disp } \\
(\mathrm{mm})\end{array}$ \\
\hline \multirow[t]{3}{*}{ FF1 } & FB & 1.37 & 0.18 & 17.5 & 3.4 & \\
\hline & BI & 1.00 & 0.36 & 42.6 & 27.4 & 16.7 \\
\hline & $\%$ reduction & 26.96 & -98.15 & -143.43 & & \\
\hline \multirow[t]{3}{*}{ FF2 } & FB & 2.52 & 0.47 & 44.6 & 8.5 & \\
\hline & BI & 1.31 & 0.61 & 72.9 & 56.3 & 44.2 \\
\hline & $\%$ reduction & 48.03 & -27.82 & -63.45 & & \\
\hline \multirow[t]{3}{*}{ FF3 } & $\mathrm{FB}$ & 1.54 & 0.28 & 24.6 & 4.9 & \\
\hline & BI & 1.04 & 0.34 & 39.2 & 25.2 & 15 \\
\hline & $\%$ reduction & 32.60 & -20.00 & -59.35 & & \\
\hline \multirow{3}{*}{ FF4 } & FB & 1.94 & 0.27 & 24.2 & 4.8 & \\
\hline & BI & 0.90 & 0.37 & 38.8 & 23.9 & 12.9 \\
\hline & $\%$ reduction & 53.61 & -34.15 & -60.33 & & \\
\hline \multirow[t]{3}{*}{ FF5 } & FB & 1.79 & 0.30 & 24 & 5.5 & \\
\hline & BI & 1.25 & 0.38 & 51 & 31.2 & 19.7 \\
\hline & $\%$ reduction & 30.13 & -29.21 & -112.50 & & \\
\hline \multirow[t]{3}{*}{ FF6 } & FB & 1.79 & 0.28 & 24.7 & 4.9 & \\
\hline & BI & 1.05 & 0.35 & 40 & 25.5 & 15 \\
\hline & $\%$ reduction & 41.64 & -26.51 & -61.94 & & \\
\hline \multirow[t]{3}{*}{ NFD1 } & FB & 1.86 & 0.29 & 25.3 & 6.2 & \\
\hline & $\mathrm{BI}$ & 1.477 & 0.40 & 82.1 & 70 & 57.9 \\
\hline & $\%$ reduction & 20.63 & -37.50 & -224.51 & & \\
\hline \multirow[t]{3}{*}{ NFD2 } & FB & 2.05 & 0.41 & 38.6 & 7.3 & \\
\hline & $\mathrm{BI}$ & 1.266 & 0.30 & 59.8 & 50.4 & 41.3 \\
\hline & $\%$ reduction & 38.27 & 26.02 & -54.92 & & \\
\hline \multirow[t]{3}{*}{ NFD3 } & FB & 1.81 & 0.35 & 32.9 & 6 & \\
\hline & BI & 1.15 & 0.16 & 46.1 & 41.6 & 36.8 \\
\hline & $\%$ reduction & 36.63 & 53.85 & -40.12 & & \\
\hline \multirow[t]{3}{*}{ NFD4 } & FB & 1.45 & 0.21 & 21.2 & 4 & \\
\hline & BI & 1.30 & 0.28 & 44.7 & 36.5 & 28.1 \\
\hline & $\%$ reduction & 10.81 & -35.48 & -110.85 & & \\
\hline \multirow[t]{3}{*}{ NFD5 } & $\mathrm{FB}$ & 1.90 & 0.31 & 23.6 & 5.7 & \\
\hline & BI & 1.40 & 0.23 & 45.3 & 38.6 & 31.8 \\
\hline & $\%$ reduction & 26.47 & 26.09 & -91.95 & & \\
\hline \multirow[t]{3}{*}{ NFD6 } & FB & 2.10 & 0.31 & 27.6 & 6.2 & \\
\hline & BI & 1.27 & 0.31 & 51.9 & 42.6 & 33.4 \\
\hline & $\%$ reduction & 39.34 & 2.13 & -88.04 & & \\
\hline \multirow[t]{3}{*}{ NFS1 } & $\mathrm{FB}$ & 2.01 & 0.29 & 28.1 & 5.6 & \\
\hline & BI & 1.46 & 0.33 & 59.3 & 48.8 & 38.8 \\
\hline & $\%$ reduction & 27.44 & -14.94 & -111.03 & & \\
\hline \multirow[t]{3}{*}{ NFS2 } & $\mathrm{FB}$ & 2.04 & 0.39 & 32.9 & 7.2 & \\
\hline & BI & 1.45 & 0.40 & 63.9 & 51.7 & 39.8 \\
\hline & $\%$ reduction & 29.07 & -0.85 & -94.22 & & \\
\hline \multirow[t]{3}{*}{ NFS3 } & FB & 2.10 & 0.22 & 19.2 & 4.1 & \\
\hline & BI & 1.23 & 0.19 & 36.3 & 30.2 & 24.5 \\
\hline & $\%$ reduction & 41.70 & 12.31 & -89.06 & & \\
\hline \multirow[t]{3}{*}{ NFS4 } & FB & 1.46 & 0.23 & 18.4 & 4.1 & \\
\hline & BI & 1.19 & 0.26 & 38 & 30.2 & 22.4 \\
\hline & $\%$ reduction & 18.71 & -13.04 & -106.52 & & \\
\hline \multirow[t]{3}{*}{ NFS5 } & $\mathrm{FB}$ & 2.19 & 0.28 & 25.1 & 5.3 & \\
\hline & BI & 1.43 & 0.21 & 48.2 & 41.6 & 35.4 \\
\hline & $\%$ reduction & 34.81 & 26.19 & -92.03 & & \\
\hline \multirow[t]{3}{*}{ NFS6 } & $\mathrm{FB}$ & 2.07 & 0.25 & 19.3 & 4.5 & \\
\hline & $\mathrm{BI}$ & 1.23 & 0.23 & 39.8 & 32.1 & 25.3 \\
\hline & $\%$ reduction & 40.55 & 9.33 & -106.22 & & \\
\hline
\end{tabular}


Table 6. Eight storey response parameters

\begin{tabular}{|c|c|c|c|c|c|c|}
\hline Earthquake & Type of structure & $\begin{array}{c}\text { Roof accel } \\
\left(\mathrm{m} / \mathrm{s}^{2}\right)\end{array}$ & $\begin{array}{c}\text { Max Drift ratio } \\
(\%)\end{array}$ & $\begin{array}{c}\text { Roof disp } \\
(\mathrm{mm})\end{array}$ & $\begin{array}{l}\text { first floor disp } \\
(\mathrm{mm})\end{array}$ & $\begin{array}{c}\text { Isolator disp } \\
(\mathrm{mm})\end{array}$ \\
\hline \multirow[t]{3}{*}{ FF1 } & FB & 0.95 & 0.36 & 58.2 & 4.6 & \\
\hline & BI & 0.70 & 0.24 & 66.6 & 47.2 & 40 \\
\hline & $\%$ reduction & 26.15 & 32.71 & -14.43 & & \\
\hline \multirow[t]{3}{*}{ FF2 } & FB & 1.49 & 0.42 & 70.8 & 5.1 & \\
\hline & $\mathrm{BI}$ & 0.72 & 0.29 & 101.4 & 78.1 & 69.3 \\
\hline & $\%$ reduction & 51.61 & 29.60 & -43.22 & & \\
\hline \multirow[t]{3}{*}{ FF3 } & $\mathrm{FB}$ & 0.91 & 0.43 & 69.6 & 5.8 & \\
\hline & $\mathrm{BI}$ & 0.68 & 0.39 & 81 & 50.9 & 39.2 \\
\hline & $\%$ reduction & 25.24 & 10.00 & -16.38 & & \\
\hline \multirow{3}{*}{ FF4 } & $\mathrm{FB}$ & 0.94 & 0.40 & 65.9 & 5.3 & \\
\hline & BI & 0.69 & 0.29 & 66.7 & 43.8 & 35.2 \\
\hline & $\%$ reduction & 25.99 & 28.33 & -1.21 & & \\
\hline \multirow[t]{3}{*}{ FF5 } & FB & 1.07 & 0.47 & 78.1 & 6.2 & \\
\hline & $\mathrm{BI}$ & 0.74 & 0.37 & 85.9 & 57.1 & 46 \\
\hline & $\%$ reduction & 31.18 & 21.28 & -9.99 & & \\
\hline \multirow[t]{3}{*}{ FF6 } & FB & 0.94 & 0.35 & 56.7 & 4.6 & \\
\hline & $\mathrm{BI}$ & 0.67 & 0.21 & 59.7 & 37.4 & 31.2 \\
\hline & $\%$ reduction & 28.32 & 40.38 & -5.29 & & \\
\hline \multirow{3}{*}{ NFD1 } & FB & 1.42 & 0.74 & 121 & 9.9 & \\
\hline & $\mathrm{BI}$ & 0.89 & 0.63 & 203.1 & 153.9 & 135.1 \\
\hline & $\%$ reduction & 37.10 & 15.32 & -67.85 & & \\
\hline \multirow[t]{3}{*}{ NFD2 } & FB & 1.26 & 0.84 & 137.3 & 11.1 & \\
\hline & $\mathrm{BI}$ & 1.00 & 0.72 & 256.4 & 199.4 & 177.8 \\
\hline & $\%$ reduction & 20.62 & 14.29 & -86.74 & & \\
\hline \multirow[t]{3}{*}{ NFD3 } & FB & 1.02 & 0.39 & 65.9 & 5.3 & \\
\hline & BI & 0.67 & 0.34 & 74.3 & 46.8 & 36.6 \\
\hline & $\%$ reduction & 34.38 & 12.82 & -12.75 & & \\
\hline \multirow{3}{*}{ NFD4 } & FB & 1.17 & 0.56 & 90.4 & 7.3 & \\
\hline & BI & 0.79 & 0.41 & 109.3 & 76.9 & 64.5 \\
\hline & $\%$ reduction & 32.25 & 25.75 & -20.91 & & \\
\hline \multirow[t]{3}{*}{ NFD5 } & $\mathrm{FB}$ & 1.06 & 0.37 & 60 & 5.1 & \\
\hline & BI & 0.76 & 0.25 & 56.7 & 35.7 & 28.2 \\
\hline & $\%$ reduction & 28.59 & 32.43 & 5.50 & & \\
\hline \multirow[t]{3}{*}{ NFD6 } & FB & 1.31 & 0.55 & 91.7 & 7.4 & \\
\hline & BI & 0.78 & 0.34 & 100.2 & 73.2 & 62.9 \\
\hline & $\%$ reduction & 40.63 & 37.58 & -9.27 & & \\
\hline \multirow[t]{3}{*}{ NFS1 } & $\mathrm{FB}$ & 1.36 & 0.59 & 99.2 & 7.6 & \\
\hline & $\mathrm{BI}$ & 0.89 & 0.47 & 135.1 & 97.6 & 83.6 \\
\hline & $\%$ reduction & 34.10 & 21.35 & -36.19 & & \\
\hline \multirow[t]{3}{*}{ NFS2 } & FB & 1.20 & 0.62 & 102.7 & 8.3 & \\
\hline & BI & 0.82 & 0.49 & 127 & 88.8 & 74.2 \\
\hline & $\%$ reduction & 31.39 & 21.08 & -23.66 & & \\
\hline \multirow[t]{3}{*}{ NFS3 } & FB & 0.92 & 0.38 & 61.9 & 5.1 & \\
\hline & BI & 0.68 & 0.33 & 64.5 & 37.9 & 27.9 \\
\hline & $\%$ reduction & 26.47 & 13.04 & -4.20 & & \\
\hline \multirow[t]{3}{*}{ NFS4 } & FB & 1.11 & 0.45 & 75.5 & 6 & \\
\hline & BI & 0.77 & 0.30 & 77.6 & 53.5 & 44.5 \\
\hline & $\%$ reduction & 30.87 & 33.82 & -2.78 & & \\
\hline \multirow[t]{3}{*}{ NFS5 } & $\mathrm{FB}$ & 1.03 & 0.35 & 58.9 & 4.3 & \\
\hline & BI & 0.76 & 0.29 & 67.8 & 45.1 & 36.5 \\
\hline & $\%$ reduction & 26.18 & 18.87 & -15.11 & & \\
\hline \multirow[t]{3}{*}{ NFS6 } & $\mathrm{FB}$ & 0.99 & 0.49 & 80.3 & 6.3 & \\
\hline & $\mathrm{BI}$ & 0.74 & 0.41 & 96.3 & 64 & 51.6 \\
\hline & $\%$ reduction & 24.96 & 15.65 & -19.93 & & \\
\hline
\end{tabular}


Table 7. Twelve storey response parameters

\begin{tabular}{|c|c|c|c|c|c|c|}
\hline Earthquake & Type of structure & $\begin{array}{c}\text { Roof accel } \\
\left(\mathrm{m} / \mathrm{s}^{2}\right)\end{array}$ & $\begin{array}{c}\text { Max Drift ratio } \\
(\%)\end{array}$ & $\begin{array}{c}\text { Roof disp } \\
(\mathrm{mm})\end{array}$ & $\begin{array}{l}\text { first floor disp } \\
(\mathrm{mm})\end{array}$ & $\begin{array}{c}\text { Isolator disp } \\
(\mathrm{mm})\end{array}$ \\
\hline \multirow[t]{3}{*}{ FF1 } & FB & 0.64 & 0.38 & 92.4 & 3.6 & \\
\hline & BI & 0.52 & 0.22 & 75.6 & 32.8 & 26.6 \\
\hline & $\%$ reduction & 18.03 & 41.59 & 18.18 & & \\
\hline \multirow[t]{3}{*}{ FF2 } & FB & 0.72 & 0.39 & 91.8 & 4 & \\
\hline & $\mathrm{BI}$ & 0.55 & 0.32 & 89.6 & 45.8 & 36.1 \\
\hline & $\%$ reduction & 22.82 & 17.09 & 2.40 & & \\
\hline \multirow[t]{3}{*}{ FF3 } & $\mathrm{FB}$ & 0.78 & 0.65 & 155.1 & 6.4 & \\
\hline & BI & 0.57 & 0.47 & 133.8 & 69.9 & 55.8 \\
\hline & $\%$ reduction & 26.60 & 27.32 & 13.73 & & \\
\hline \multirow{3}{*}{ FF4 } & FB & 0.71 & 0.39 & 92.8 & 4.1 & \\
\hline & BI & 0.59 & 0.27 & 77.7 & 36.5 & 28.3 \\
\hline & $\%$ reduction & 16.61 & 29.31 & 16.27 & & \\
\hline \multirow[t]{3}{*}{ FF5 } & FB & 0.75 & 0.52 & 129 & 5 & \\
\hline & $\mathrm{BI}$ & 0.60 & 0.40 & 113.3 & 58.4 & 46.4 \\
\hline & $\%$ reduction & 20.52 & 23.08 & 12.17 & & \\
\hline \multirow[t]{3}{*}{ FF6 } & FB & 0.67 & 0.37 & 90.8 & 4 & \\
\hline & $\mathrm{BI}$ & 0.56 & 0.28 & 76.8 & 37.8 & 29.5 \\
\hline & $\%$ reduction & 16.45 & 25.23 & 15.42 & & \\
\hline \multirow{3}{*}{ NFD1 } & FB & 1.46 & 1.37 & 350.2 & 14.1 & \\
\hline & $\mathrm{BI}$ & 0.85 & 0.86 & 367.6 & 248.9 & 223.1 \\
\hline & $\%$ reduction & 41.79 & 37.38 & -4.97 & & \\
\hline \multirow[t]{3}{*}{ NFD2 } & FB & 1.58 & 1.64 & 409.9 & 15.8 & \\
\hline & $\mathrm{BI}$ & 0.92 & 0.92 & 413.2 & 284.4 & 256.7 \\
\hline & $\%$ reduction & 41.83 & 43.81 & -0.81 & & \\
\hline \multirow[t]{3}{*}{ NFD3 } & FB & 0.70 & 0.55 & 133.6 & 5.3 & \\
\hline & BI & 0.55 & 0.43 & 122.2 & 63.8 & 50.8 \\
\hline & $\%$ reduction & 22.00 & 20.73 & 8.53 & & \\
\hline \multirow[t]{3}{*}{ NFD4 } & FB & 0.86 & 0.63 & 157.5 & 5.9 & \\
\hline & BI & 0.64 & 0.46 & 140.7 & 76.4 & 62.7 \\
\hline & $\%$ reduction & 25.25 & 27.89 & 10.67 & & \\
\hline \multirow[t]{3}{*}{ NFD5 } & $\mathrm{FB}$ & 0.77 & 0.48 & 112.6 & 5.2 & \\
\hline & $\mathrm{BI}$ & 0.55 & 0.36 & 91.7 & 44.7 & 33.9 \\
\hline & $\%$ reduction & 29.40 & 25.00 & 18.56 & & \\
\hline \multirow[t]{3}{*}{ NFD6 } & FB & 1.00 & 0.62 & 148.6 & 6 & \\
\hline & BI & 0.65 & 0.37 & 122.8 & 72 & 60.9 \\
\hline & $\%$ reduction & 34.77 & 40.64 & 17.36 & & \\
\hline \multirow[t]{3}{*}{ NFS1 } & $\mathrm{FB}$ & 0.76 & 0.84 & 201.3 & 8.6 & \\
\hline & $\mathrm{BI}$ & 0.69 & 0.50 & 207.4 & 123.2 & 108.2 \\
\hline & $\%$ reduction & 8.79 & 40.48 & -3.03 & & \\
\hline \multirow[t]{3}{*}{ NFS2 } & FB & 0.89 & 0.76 & 185.5 & 8.1 & \\
\hline & BI & 0.67 & 0.59 & 193 & 111.9 & 94.1 \\
\hline & $\%$ reduction & 24.69 & 21.59 & -4.04 & & \\
\hline \multirow[t]{3}{*}{ NFS3 } & FB & 0.68 & 0.55 & 134 & 5.3 & \\
\hline & BI & 0.55 & 0.43 & 110.5 & 50.4 & 37.5 \\
\hline & $\%$ reduction & 19.19 & 22.29 & 17.54 & & \\
\hline \multirow[t]{3}{*}{ NFS4 } & FB & 0.80 & 0.42 & 100.6 & 4.3 & \\
\hline & BI & 0.64 & 0.25 & 85.1 & 46.5 & 38.9 \\
\hline & $\%$ reduction & 19.84 & 39.68 & 15.41 & & \\
\hline \multirow[t]{3}{*}{ NFS5 } & $\mathrm{FB}$ & 0.64 & 0.38 & 93.4 & 3.6 & \\
\hline & BI & 0.60 & 0.28 & 78.9 & 38.6 & 30.2 \\
\hline & $\%$ reduction & 6.23 & 26.32 & 15.52 & & \\
\hline \multirow[t]{3}{*}{ NFS6 } & FB & 0.75 & 0.65 & 160.7 & 6.3 & \\
\hline & $\mathrm{BI}$ & 0.60 & 0.50 & 143 & 73.6 & 58.6 \\
\hline & $\%$ reduction & 19.71 & 23.47 & 11.01 & & \\
\hline
\end{tabular}


Table 8. Four storey base shear ratios $V_{b}(N L T H A) / V_{b}(D D B D)$

\begin{tabular}{|c|c|c|c|c|c|c|c|c|c|c|c|}
\hline & & & \multicolumn{3}{|c|}{ Far field } & \multicolumn{3}{|c|}{ Near field forward directivity } & \multicolumn{3}{|c|}{ Near field fling step } \\
\hline & $\mathrm{V}_{\mathrm{b}}{ }^{(\mathrm{a})}$ & & & $\mathrm{V}_{\mathrm{b}}{ }^{(\mathrm{b})}$ & (b)/(a) & & $\mathrm{V}_{\mathrm{b}}{ }^{(\mathrm{c})}$ & (c)/(a) & & $\mathrm{V}_{\mathrm{b}}^{(\mathrm{d})}$ & (d)/(a) \\
\hline \multirow{6}{*}{$\begin{array}{c}\text { BI } \\
\text { DDBD }\end{array}$} & \multirow{6}{*}{1237} & \multirow{6}{*}{$\begin{array}{c}\text { BI } \\
\text { Time } \\
\text { history }\end{array}$} & FF1 & 942 & 0.76 & NFD1 & 1541 & 1.25 & NFS1 & 1372 & 1.11 \\
\hline & & & FF2 & 1088 & 0.88 & NFD2 & 1428 & 1.15 & NFS2 & 1481 & 1.20 \\
\hline & & & FF3 & 941 & 0.76 & NFD3 & 1016 & 0.82 & NFS3 & 1037 & 0.84 \\
\hline & & & FF4 & 970 & 0.78 & NFD4 & 1251 & 1.01 & NFS4 & 1172 & 0.95 \\
\hline & & & FF5 & 885 & 0.72 & NFD5 & 1173 & 0.95 & NFS5 & 1198 & 0.97 \\
\hline & & & FF6 & 943 & 0.76 & NFD6 & 1268 & 1.02 & NFS6 & 1232 & 1.00 \\
\hline \multirow{6}{*}{$\begin{array}{c}\text { FB } \\
\text { DDBD }\end{array}$} & \multirow{6}{*}{3524} & \multirow{6}{*}{$\begin{array}{c}\text { FB } \\
\text { Time } \\
\text { history }\end{array}$} & FF1 & 1216 & 0.35 & NFD1 & 1973 & 0.56 & NFS1 & 1802 & 0.51 \\
\hline & & & FF2 & 1906 & 0.54 & NFD2 & 1661 & 0.47 & NFS2 & 1692 & 0.48 \\
\hline & & & FF3 & 1213 & 0.34 & NFD3 & 1432 & 0.41 & NFS3 & 1285 & 0.36 \\
\hline & & & FF4 & 1190 & 0.34 & NFD4 & 1501 & 0.43 & NFS4 & 1328 & 0.38 \\
\hline & & & FF5 & 1397 & 0.40 & NFD5 & 1438 & 0.41 & NFS5 & 1536 & 0.44 \\
\hline & & & FF6 & 1217 & 0.35 & NFD6 & 1519 & 0.43 & NFS6 & 1303 & 0.37 \\
\hline
\end{tabular}

Table 9. Eight storey base shear ratios $V_{b}(N L T H A) / V_{b}(D D B D)$

\begin{tabular}{|c|c|c|c|c|c|c|c|c|c|c|c|}
\hline & & & \multicolumn{3}{|c|}{ Far field } & \multicolumn{3}{|c|}{ Near field forward directivity } & \multicolumn{3}{|c|}{ Near field fling step } \\
\hline & \multicolumn{2}{|l|}{$\mathrm{V}_{\mathrm{b}}^{(\mathrm{a})}$} & & $\mathrm{V}_{\mathrm{b}}{ }^{(\mathrm{b})}$ & (b)/(a) & & $\mathrm{V}_{\mathrm{b}}{ }^{(\mathrm{c})}$ & (c)/(a) & & $\mathrm{V}_{\mathrm{b}}^{(\mathrm{d})}$ & (d)/(a) \\
\hline \multirow{6}{*}{$\begin{array}{c}\text { BI } \\
\text { DDBD }\end{array}$} & \multirow{6}{*}{2621} & \multirow{6}{*}{$\begin{array}{c}\text { BI } \\
\text { Time } \\
\text { history }\end{array}$} & FF1 & 1724 & 0.66 & NFD1 & 2769 & 1.06 & NFS1 & 2277 & 0.87 \\
\hline & & & FF2 & 1835 & 0.70 & NFD2 & 3120 & 1.19 & NFS2 & 2259 & 0.86 \\
\hline & & & FF3 & 1973 & 0.75 & NFD3 & 1852 & 0.71 & NFS3 & 1782 & 0.68 \\
\hline & & & FF4 & 1743 & 0.66 & NFD4 & 2148 & 0.82 & NFS4 & 1797 & 0.69 \\
\hline & & & FF5 & 1980 & 0.76 & NFD5 & 1674 & 0.64 & NFS5 & 1737 & 0.66 \\
\hline & & & FF6 & 1722 & 0.66 & NFD6 & 1930 & 0.74 & NFS6 & 1927 & 0.74 \\
\hline \multirow{6}{*}{$\begin{array}{c}\text { FB } \\
\text { DDBD }\end{array}$} & \multirow{6}{*}{3554} & \multirow{6}{*}{$\begin{array}{c}\text { FB } \\
\text { Time } \\
\text { history }\end{array}$} & FF1 & 1776 & 0.50 & NFD1 & 2961 & 0.83 & NFS1 & 2372 & 0.67 \\
\hline & & & FF2 & 2052 & 0.58 & NFD2 & 3317 & 0.93 & NFS2 & 2563 & 0.72 \\
\hline & & & FF3 & 2074 & 0.58 & NFD3 & 2036 & 0.57 & NFS3 & 1979 & 0.56 \\
\hline & & & FF4 & 2030 & 0.57 & NFD4 & 2357 & 0.66 & NFS4 & 2151 & 0.61 \\
\hline & & & FF5 & 2165 & 0.61 & NFD5 & 1894 & 0.53 & NFS5 & 1715 & 0.48 \\
\hline & & & FF6 & 1814 & 0.51 & NFD6 & 2423 & 0.68 & NFS6 & 2144 & 0.60 \\
\hline
\end{tabular}

Table 10. Twelve storey base shear ratios $V_{b}(N L T H A) / V_{b}(D D B D)$

\begin{tabular}{|c|c|c|c|c|c|c|c|c|c|c|c|}
\hline & & & \multicolumn{3}{|c|}{ Far field } & \multicolumn{3}{|c|}{ Near field forward directivity } & \multicolumn{3}{|c|}{ Near field fling step } \\
\hline & \multicolumn{2}{|l|}{$\mathrm{V}_{\mathrm{b}}^{(\mathrm{a})}$} & & $\mathrm{V}_{\mathrm{b}}{ }^{(\mathrm{b})}$ & (b)/(a) & & $\mathrm{V}_{\mathrm{b}}{ }^{(\mathrm{c})}$ & (c)/(a) & & $\mathrm{V}_{\mathrm{b}}^{(\mathrm{d})}$ & (d)/(a) \\
\hline \multirow{6}{*}{$\begin{array}{c}\text { BI } \\
\text { DDBD }\end{array}$} & \multirow{6}{*}{4095} & \multirow{6}{*}{$\begin{array}{l}\text { BI Time } \\
\text { history }\end{array}$} & FF1 & 2053 & 0.50 & NFD1 & 4114 & 1.00 & NFS1 & 2937 & 0.72 \\
\hline & & & FF2 & 2025 & 0.49 & NFD2 & 4397 & 1.07 & NFS2 & 2986 & 0.73 \\
\hline & & & FF3 & 2636 & 0.64 & NFD3 & 2493 & 0.61 & NFS3 & 2432 & 0.59 \\
\hline & & & FF4 & 2015 & 0.49 & NFD4 & 2670 & 0.65 & NFS4 & 1997 & 0.49 \\
\hline & & & FF5 & 2405 & 0.59 & NFD5 & 2181 & 0.53 & NFS5 & 1934 & 0.47 \\
\hline & & & FF6 & 2094 & 0.51 & NFD6 & 2444 & 0.60 & NFS6 & 2691 & 0.66 \\
\hline \multirow{6}{*}{$\begin{array}{c}\text { FB } \\
\text { DDBD }\end{array}$} & \multirow{6}{*}{4136} & \multirow{6}{*}{$\begin{array}{l}\text { FB Time } \\
\text { history }\end{array}$} & FF1 & 1712 & 0.41 & NFD1 & 4767 & 1.15 & NFS1 & 3329 & 0.81 \\
\hline & & & FF2 & 1980 & 0.48 & NFD2 & 5406 & 1.31 & NFS2 & 3224 & 0.78 \\
\hline & & & FF3 & 2705 & 0.65 & NFD3 & 2327 & 0.56 & NFS3 & 2395 & 0.58 \\
\hline & & & FF4 & 2186 & 0.53 & NFD4 & 2649 & 0.64 & NFS4 & 2190 & 0.53 \\
\hline & & & FF5 & 2420 & 0.59 & NFD5 & 2501 & 0.60 & NFS5 & 1671 & 0.40 \\
\hline & & & FF6 & 2045 & 0.49 & NFD6 & 2809 & 0.68 & NFS6 & 2606 & 0.63 \\
\hline
\end{tabular}



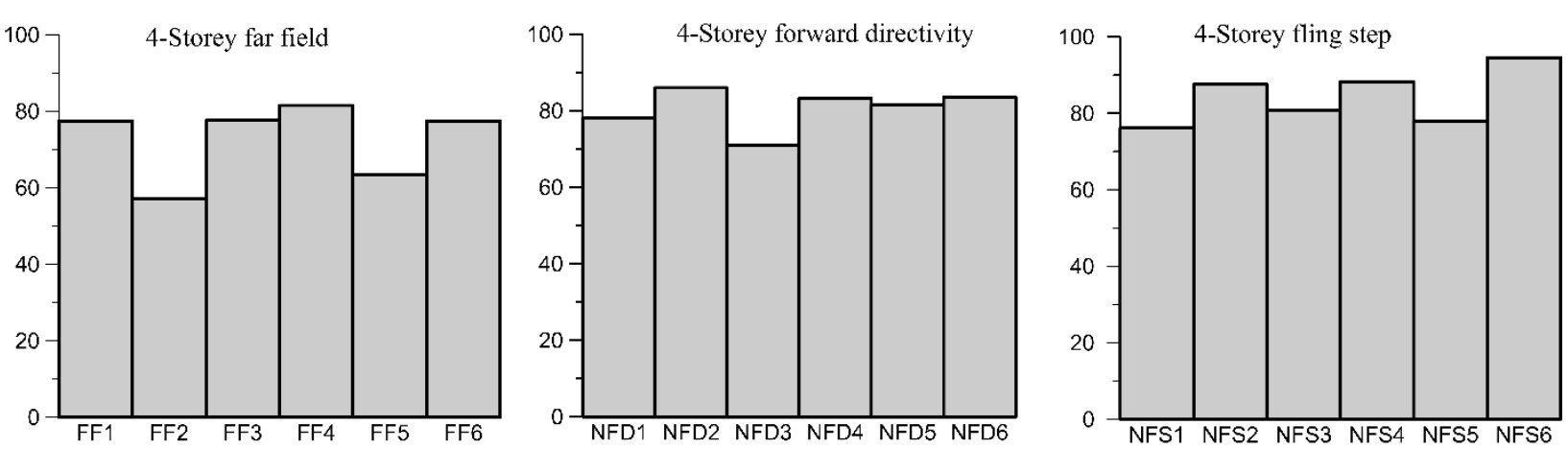

Figure 7. Four storey base shear ratio of fixed base vs base-isolated structure of NLTHA
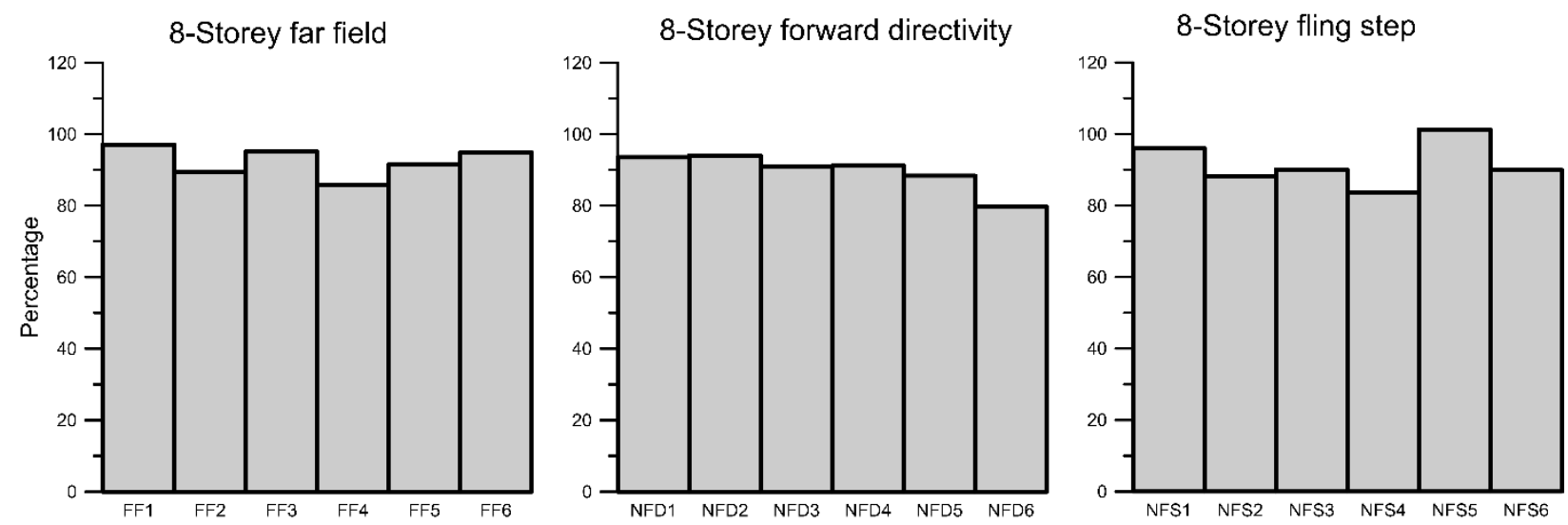

Figure 8. Eight storey base shear ratio of fixed base vs base-isolated structure of NLTHA
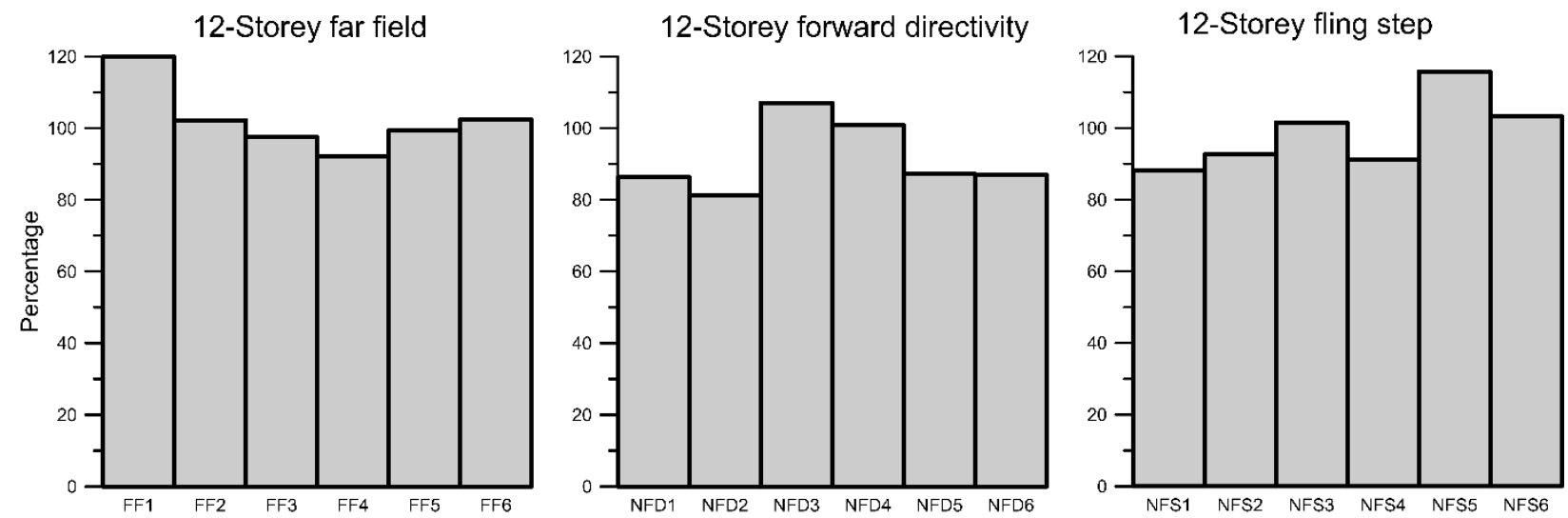

Figure 9. Twelve storey base shear ratio of fixed base vs base-isolated structure of NLTHA

\section{DISCUSSION OF THE RESULTS}

As observed in Tables $4-7$, the horizontal stiffness of the seismic isolator is low, thereby the time period of a seismically isolated structure increases which in turn results in reduced base shear. The damping of the system also increases by using the isolators. Interstorey drift ratio is very much less than the convention building since the displacements are concentrated at the level of isolators. Since the floor acceleration is reduced by using the isolators, acceleration sensitive equipment in the important buildings are not much damaged. 
As seen in Table 4, the difference in time period decreases with increase in height of the structure of the base-isolated structure compared to the fixed base [26]. This increases the stiffness which in turn increases the base shear in tall base-isolated structure as seen in Figures 7- 9 which also indicates that higher mode factor should be taken into account [8]. The demand parameters increase significantly for near-fault ground motion compared to far-field motion despite the lower value of peak ground acceleration of near-fault ground motion as observed from Table 5 to Table 10 for all the buildings considered [36].

The following observations are made in the study:

1. In the present procedure, $\mathrm{V}_{\mathrm{b}(\mathrm{NLTHA})}$ is within $\mathrm{V}_{\mathrm{b}(\mathrm{DDBD})}$ for both $\mathrm{FB}$ (fixed base) and $\mathrm{BI}$ (base-isolated) structure for far field ground motions (Tables 8 - 10).

2. $\mathrm{V}_{\mathrm{b}(\mathrm{NLTHA})}$ for 4-storey building exceeds $\mathrm{V}_{\mathrm{b} \text { (DDBD) }}$ up to $25 \%$ in near field forward directivity (NFD 1, NFD 2, NFD 4, NFD 6) and up to $20 \%$ in case of near field fling step (NFS 1, NFS 2) for the BI structure (Table 8).

3. For 8-storey BI, $\mathrm{V}_{\mathrm{b}(\mathrm{NLTHA})}$ exceeds $\mathrm{V}_{\mathrm{b}(\mathrm{DDBD})}$ up to $19 \%$ in the case of near field forward directivity (NFD 1, NFD 2) (Table 9).

4. For 12-storey $\mathrm{BI}, \mathrm{V}_{\mathrm{b}(\mathrm{NLTHA})}$ exceeds $\mathrm{V}_{\mathrm{b}(\mathrm{DDBD})}$ up to $7 \%$ in case of near field forward directivity (NFD 2) and $31 \%$ for near field fling step (NFD 1, NFD 2) for FB structure (Table 10).

5. $\mathrm{V}_{\mathrm{b} \text { (NLTHA) }}$ of $\mathrm{BI}$ structure is greater than $\mathrm{V}_{\mathrm{b} \text { (NLTHA) }}$ of FB for 12-storey building as well as $\mathrm{V}_{\mathrm{b}(\mathrm{DDBD})}$ for $\mathrm{BI}$ is nearly equal to $\mathrm{V}_{\mathrm{b}(\mathrm{DDBD})}$ for $\mathrm{FB}$ (Table 10 ).

6. The base shear values of BI buildings in case of nonlinear time history analysis were less than the corresponding fixed support values for four storey (Figure 7) and except one case in fling step, NFS 5 in eight storey (Figure 8). But for 12 storey building exceeds the base shear values of FB (Figure 9) for the far field, near field forward directivity and near field fling step ground motions.

7. The equivalent damping in the structure with FB is the same for all the buildings since the drift limit is $2 \%$ and the plan configuration is the same (Table 4).

8. For the 4-storey building, the drift ratios of BI structure were higher in all the far field, 2 cases in forward directivity (NFD 1, NFD 4), 3 cases in fling step (NFS 1, NFS 2, NFS 4) ground motions (Table 5) when compared to FB.

9. Isolator displacement values were within the design displacement values (Tables 5 - 7).

10. There is a reduced top floor acceleration in BI buildings when compared to FB (Tables 5 - 7).

11. There is not much difference in base shear of four and eight storey fixed building, since the mode shape expression does not vary according to the height of the building as defined by Priestley et al. [8].

\section{CONCLUSIONS}

The Direct displacement-based design developed by Cardone et al was applied to four, eight and twelve storey buildings regular in the plan for the fixed base and lead rubber bearing as isolators, subjected to the far field, near field directivity and near field fling step ground motions. A total of eighteen ground motions, six in each type of ground motions were considered. The following conclusions were drawn from the study:

1. The method proposed by Cardone et al holds good for the far field ground motions with fixed support as well as the base-isolated buildings frames. The base shear accounts for a maximum of $76 \%, 76 \%$ and $64 \%$ for base-isolated buildings and 54\%, 58\% and 64\% for fixed support four, eight and twelve storey buildings respectively.

2. The base shear demand was higher for the near field forward directivity and near field fling step method as seen by nonlinear time history analysis.

3. There was significant reduction in drift ratios in eight and twelve storey base-isolated buildings and the percentage increased for four storey although the values are very much less than the design drift of $2 \%$. The maximum drift was predominantly in the first storey in four storey structure.

4. The reduced floor acceleration in the base-isolated building indicates the safety and comfort of the occupants. 
5. Although this study proves the applicability of the method to far field ground motions, suggest that still more earthquake ground motions be considered especially for near field directivity and near field fling step method to possibly finding a modification factor.

\section{CONFLICTS OF INTEREST}

No conflict of interest was declared by the authors.

\section{REFERENCES}

[1] Shibata, A., Sozen, M. A., "Substitute-Structure Method for Seismic Design in R/C", American Society of Civil Eengineers Journal of the Structral Division, 102(1): 1-18, (1976).

[2] Applied Technology Council 40, "Seismic Evaluation and Retrofit of Concrete Buildings", (1): (1996).

[3] Federal Emergency Management Agency 273, "NEHRP guidelines for the seismic rehabilitation of buildings", (1997).

[4] Federal Emergency Management Agency 356, "Prestandard and Commentary for the Seismic Rehabilitation of Buildings", (2000).

[5] Structural Engineers Association of California, Blue Book., "Recommended Lateral Force Requirements and Commentary", (1999).

[6] Federation Internationale du Beton Bulletin 25, "Displacement-based seismic design of reinforced concrete buildings", (2003).

[7] Eurocode 8, "Design of structures for earthquake resistance —Part 1: General rules, seismic actions and rules for buildings", (July 2009): (2004).

[8] Priestley, M.J.N., Pettinga, J.D., "Dynamic Behaviour of Reinforced Concrete Frames Designed With Direct Displacement-Based Design", Journal of Earthquake Engineering, 9: 309-330, (2005).

[9] Malekpour, S., Dashti, F., "Application of the Direct Displacement Based Design Methodology for Different Types of RC Structural Systems", International Journal of Concrete Structures and Materials, 7(2): 135-153, (2013).

[10] Vidot-Vega, A.L., Kowalsky, M.J., "Drift, strain limits and ductility demands for RC moment frames designed with displacement-based and force-based design methods", Engineering Structures, 51: 128-140, (2013).

[11] Abebe, B.H., Lee, J.S., "Extension of Direct Displacement-Based Design to Include Higher Mode Effects in Planar Reinforced Concrete Frame Buildings", Journal of Earthquake Engineering Society of Korea, 22(5): 299-309, (2018).

[12] Sullivan, T.J., Lago, A., "Towards a simplified Direct DBD procedure for the seismic design of moment resisting frames with viscous dampers", Engineering Structures, 35: 140-148, (2012).

[13] Ayala, G., Castellanos, H., Lopez, S., "A displacement-based seismic design method with damage control for RC buildings", Earthquakes and Structures, 3(3): 413-434, (2012). 
[14] Sheth, R., Prajapati, J., Soni, D., "Comparative study nonlinear static pushover analysis and displacement based adaptive pushover analysis method", International Journal of Structural Engineering, 9(1): 81-90, (2018).

[15] Afarani, M.H.C., Nicknam, A., "Seismic Response of Mass Irregular Steel Moment Resisting Frames (SMRF) according to performance levels from IDA approach", Gazi University Journal of Science, 25(3): 751-760, (2012).

[16] Bhandari, M., Bharti, S.D., Shrimali, M.K., Datta, T.K., "Seismic Fragility Analysis of BaseIsolated Building Frames Excited by Near- and Far-Field Earthquakes", Journal of Performance of Constructed Facilities, 33(3): 1-16, (2019).

[17] Kalkan, E., Kunnath, S.K., "Effects of fling step and forward directivity on seismic response of buildings", Earthquake Spectra, 22(2): 367-390, (2006).

[18] Moghim, F., Saadatpour, M.M., "The Applicability Of Direct Displacement-Based Design In Designing Concrete Buildings Located In Near-Fault Regions", in 14th World Conference on Earthquake Engineering, (2008).

[19] Kara, E.K., Durukan, K., "The Statistical Analysis of the Earthquake Hazard for Turkey by Generalized Linear Models", Gazi University Journal of Science, 30(4): 584-597, (2017).

[20] Sayani, P.J., Keri, L., Ryan, M., "Comparative Evaluation of Base-Isolated and Fixed-Base Buildings Using a Comprehensive Response Index", Journal of Structural Engineering, 135(June): 698-707, (2009).

[21] Bhagat, S., Wijeyewickrema, A.C., Subedi, N., "Influence of Near-Fault Ground Motions with Fling-Step and Forward-Directivity Characteristics on Seismic Response of Base-Isolated Buildings", Journal of Earthquake Engineering, 25(3): 455-474, (2021).

[22] Bhandari, M., Bharti, S.D., Shrimali, M.K., Datta, T.K., "The Numerical Study of Base-Isolated Buildings Under Near-Field and Far-Field Earthquakes", Journal of Earthquake Engineering, 22(6): 989-1007, (2018).

[23] Adnan, A., Tiong, P.L.Y., Sunaryat, J., Ghazali, M.Z.M., Malek, K.A., "Seismic base isolation of steel frame structure by hollow rubber bearings", Gazi University Journal of Science, 24(4): 841$853,(2011)$.

[24] Mermer, A.S., Mustafa Kaya, M., Arslan, A. S., "Using seismic isolation elements to protect cylindrical steel liquid storage tanks from destructive forces of earthquakes", Gazi University Journal of Science, 25(1): 165-173, (2012).

[25] Cardone, D., Dolce, M., Palermo, G., "Direct displacement-based design of seismically isolated bridges", Bulletin of Earthquake Engineering, 7(2): 391-410, (2009).

[26] Cardone, D., Dolce, M., Palermo, G., "Direct displacement-based design of buildings with different seismic isolation systems", Journal of Earthquake Engineering, 14(2): 163-191, (2010).

[27] Muljati, I., Kusuma, A., Hindarto, F., "Direct displacement based design on moment resisting frame with out-of-plane offset of frame", Procedia Engineering, 125: 1057-1064, (2015).

[28] IS-1893, Indian Standard Criteria for Earthquake Resistant Design of Structures-Part 1 General Provisions and Buildings. Buereau of Indian Standards, New Delhi, (2016).

[29] Mageba Bridge Products, Data Sheets "Lasto®lrb", (2012). 
[30] Bridgestone Corporation, "Seicmic Isolation \& Vibration Control Products Business Department", (2017).

[31] FIP Industriale, "Lead rubber bearings Series LRB", (2016).

[32] Dynamic Isolation Systems, "Seismic Isolation For Buildings and Bridges", (2007).

[33] IS-456, Indian Standard Code of Practice for Plain and Reinforced Concrete, Buereau of Indian Standards, New Delhi, (2000).

[34] Kalkan, E., Chopra, A.K., "Practical guidelines to select and scale earthquake records for nonlinear response history analysis of structures", US Geological Survey, (2010).

[35] Datta, T.K., Seismıc Analysis of Structures., John Wiley \& Sons, Singapore, (2010).

[36] Sodha, A.H., Soni, D.P., Desai, M.K, Kumar, S., "Behavior of quintuple friction pendulum system under near-fault earthquakes", Journal of Earthquake and Tsunami, 11(5): 1-23, (2017). 